OPEN ACCESS

Edited by:

Shawn Hayley,

Carleton University, Canada

Reviewed by:

Grzegorz Kreiner,

Polish Academy of Sciences, Poland

Eva Benito,

Deutsches Zentrum Für Neurodegenerative Erkrankungen,

Germany

*Correspondence: Silvia Sánchez-Ramón ssramon@salud.madrid.org;

Florence Faure

florence.faure@curie.fr

Received: 15 October 2015 Accepted: 18 January 2016 Published: 04 February 2016

Citation:

Sánchez-Ramón S and Faure F (2016) Through the Immune Looking

Glass: A Model for Brain Memory Strategies.

Front. Cell. Neurosci. 10:17. doi: 10.3389/fncel.2016.00017

\section{Through the Immune Looking Glass: A Model for Brain Memory Strategies}

\author{
Silvia Sánchez-Ramón ${ }^{1,2 *}$ and Florence Faure ${ }^{3 *}$ \\ ${ }^{1}$ Department of Clinical Immunology and Instituto de Investigación Sanitaria San Carlos (IdISSC), Hospital Clínico San Carlos, \\ Madrid, Spain, ${ }^{2}$ Department of Microbiology I, Complutense University School of Medicine, Madrid, Spain, ${ }^{3}$ Institut National \\ de la Santé et de la Recherche Médicale U932, Institut Curie, Paris, France
}

The immune system (IS) and the central nervous system (CNS) are complex cognitive networks involved in defining the identity (self) of the individual through recognition and memory processes that enable one to anticipate responses to stimuli. Brain memory has traditionally been classified as either implicit or explicit on psychological and anatomical grounds, with reminiscences of the evolutionarily-based innate-adaptive IS responses. Beyond the multineuronal networks of the CNS, we propose a theoretical model of brain memory integrating the CNS as a whole. This is achieved by analogical reasoning between the operational rules of recognition and memory processes in both systems, coupled to an evolutionary analysis. In this new model, the hippocampus is no longer specifically ascribed to explicit memory but rather it both becomes part of the innate (implicit) memory system and tightly controls the explicit memory system. Alike the antigen presenting cells for the IS, the hippocampus would integrate transient and pseudo-specific (i.e., danger-fear) memories and would drive the formation of long-term and highly specific or explicit memories (i.e., the taste of the Proust's madeleine cake) by the more complex and recent, evolutionarily speaking, neocortex. Experimental and clinical evidence is provided to support the model. We believe that the singularity of this model's approximation could help to gain a better understanding of the mechanisms operating in brain memory strategies from a large-scale network perspective.

Keywords: hippocampus, innate and adaptive memory, implicit and explicit memory, trained immunity, recognition, immune system, central nervous system

\section{ROADS TO MEMORY AND THE SELF}

Our current state of knowledge is beginning to grasp the true essence of the evolutionarily developed processes underlying context-specific recognition and memory by the central nervous system (CNS). The CNS, and more particularly the human brain, consists of an extremely complex network of interactions among neural cells. This network coordinates all actions of the organism, intrinsically embodied with abstract thinking and high intellectual functions up to what we know as mind. Meanwhile, the immune system (IS) brings together an extensive network of cells, molecules and organs that are involved in the maintenance of the organism's integrity, conceptualized by some authors as a real neural network (Varela and Coutinho, 1989, 1991). The CNS and the IS are thus complex integrative systems, showing some intriguingly similar general functions, although at different levels of organization and complexity. Both systems are involved in defining the identity (self) of the individual (in terms of unique genetic and phenotypic attributes), together with continuing cumulative experience (history), through memory. 
Memory represents one of the most transcendent properties of the neural and immunological activity, enabling modification and anticipation of responses to stimuli, and also providing a valence or category to each response. The valences can be synthesized as predominantly negative (danger-fear cues inducing effector responses in the CNS and in the IS) or positive (action through reward and tolerance, respectively). Brain memory is the substrate of our intelligence, emotions, evoked feelings and behavior, while immune memory allows efficient clearing of pathogenic microbes and tolerance to diverse symbiotic microbial populations and to the own host cells. Both systems could also be functionally equivalent in their ultimate goal, the maintenance and survival of the organism. Their capability for information processing and proactive responsiveness in the face of uncertainty are the features that define a cognitive system, although at quite different scales.

The CNS and the IS deal with the external and internal reality by direct interaction and recognize the potential stimulus through biochemical and electromagnetic coupling interactions at quite different levels. Through these interactions both systems subsequently generate an internal representation of such reality. A sharp distinction between the CNS and the IS is therefore apparent at first sight. Neurons possess one distinctive characteristic that is their intrinsic oscillatory electromagnetic activity, basis of neural transfer of information, which renders the system able to offer immediate responses. It has been commonplace to consider memory traces as structural modifications on the strength of synaptic connections at neuronal dendritic spines in a phenomenon called long-term potentiation (LTP) and on the establishment of new neural circuitries, mostly based in individual neurons and pathways in what has been called the Neuron Doctrine (Ramón y Cajal, 1891; Hebb, 1949; Kandel et al., 2014; Nabavi et al., 2014). However, the mechanisms of how a given memory is specifically encoded at the molecular and furthermore at high order associative levels remains to be deciphered.

One could object to the analogies between the CNS and IS on the grounds of the current knowledge that such analysis could work for general mechanisms, but have limited application for the specifics of brain memory. Indeed, a major problem is the integration of logical thinking and self-awareness (consciousness) of the neural memory process, which cannot have a counterpart in the IS. Caveats issued, this approximation may provide a valuable opportunity to explain the global functioning of brain memory.

We have worked on the analogies of relationships between the general mechanisms and the subcellular events for memory processes in both systems. Analogies were coupled with an evolutionary analysis of the recognition and memory strategies. Our model generates an apparent paradox: while the terminology of IS processes has been described as a metaphor of their CNS counterparts (with terms such as recognition, learning, priming, memory, tolerance and synapse, among others); we intend here the task to build-up a model of brain memory by applying the conceptual framework of the immune memory system to the brain memory system as an approach to study emergent levels of brain neural networks as a whole (Box 1).
BOX 1 | Parallelism between general rules of the IS and the CNS.

- The IS and the CNS are complex cognitive networks safeguarding the identity (self) of the individual by means of recognition and memory mechanisms throughout lifetime.

- Shared general mechanisms and phenomena in recognition, (learning) and memory are apparent and account for the high diversity, specificity and plasticity of each system.

- Analogical reasoning between operational rules of recognition and memory in the IS and the CNS allows an approximation to some CNS' emerging and associative properties of the network.

Brain memory has traditionally been classified as either implicit or explicit on psychological and anatomical grounds, with reminiscences of the evolutionarily-based innate-adaptive IS responses. According to this model, we focus on the role of hippocampus and introduce the new concept of its role in controlling both implicit and explicit memory system. Not unlike the antigen presenting cells for the IS, the hippocampus would integrate transient and pseudo-specific (i.e., danger-fear) memories and would drive the formation of long-term and highly specific memories (i.e., the taste of the Proust's madeleine cake) by the more complex and recent, evolutionarily speaking, neocortex.

\section{REVISITING THE CLASSIFICATION OF RECOGNITION AND MEMORY STRATEGIES: WHY AN IMMUNE HYPOTHESIS?}

The existing classification of implicit and explicit brain memory sets out the concepts that underlie the whole multidisciplinary knowledge and research on this intricate yet fascinating issue. However, this classification may show some inaccuracies, due mainly to the multiple and sometimes overlapping memory systems (Willingham and Goedert, 2001; Henke, 2010), and the more descriptive than explanatory taxonomy. Here, we outline some of the most common concerns for which looking through the immune optic might assist to clarify aspects in the current brain memory model:

- Notions or biases that guide our present knowledge on CNS memory: the classification of memory types initially was, and still is today, based primarily on a psychological and neuroanatomical perspective (Reber, 2013), and thus could not necessarily align with evolutionarily gained structures. Besides, the classical evolution- and scala naturae-based terminology for the brain organization does not always accurately reflect current understanding and this may have blurred the interpretation of the function of certain brain structures (Jarvis et al., 2005).

- Accelerated parallel evolution of both IS and CNS. As complex information processing systems, the major force of natural selection pressure along evolution has targeted genes affecting immunity and nervous systems, together with reproduction (Dorus et al., 2004; Barreiro and Quintana-Murci, 2010; Barry and Mattick, 2012). 
- Shared embryogenesis phenomena in both highly integrated systems, particularly in the education on identity (self) intrauterus; similarities between double-positive thymic T-cells and neuroblasts; shared programmed-cell death (PCD) mechanisms; putative non-homologous end-joining molecules (Chun, 2001); and myeloid origin of the microglia; among others.

- Primitive elements are highly conserved and remain crucial for immune and neural responsiveness. According to Medzhitov and Janeway (2002), the major decision to respond to a particular stimulus is primarily decided by the innate IS. The same principle works for the CNS, in which the major decision to respond to a particular stimulus is determinant for survival and corresponds to the most ancient part of the brain. By contrast, the ability to specifically remember a given stimulus in both systems is found exclusively in vertebrates and not in plants or invertebrates.

- Specific (or explicit) memory is associative and remains preserved by a specialized dispersion in the complex network. The immune input contains multiple specific determinants (or epitopes) that shape their multiple cognate cells' and antibodies interactions in coordinated immune outputs. Alike, brain memories are encoded as synchronous multineuronal activation patterns and not at a single cell level (Yuste, 2015).

Additional common shared characteristics of memory processing in both systems that might show archetypal principles, are: (i) the duality of specificity and degeneracy of recognition, favoring the precision and plasticity of response; (ii) experience-dependent plasticity, as repetitive encounters reinforce the memory process inducing multiple alternative pathways for any given element; (iii) redundancy in mechanisms, more common in the innate immune and CNS for fail-safe operations, since they impact directly on survival; and (iv) feedback loops for homeostatic regulation of the system. These parallelisms have experimental evidence in pathology, as we shall illustrate.

\section{EVOLUTIONARY FRAMEWORK FOR MEMORY CLASSIFICATION}

The classification of innate and adaptive IS has been based on an evolutionary perspective, showing distinct definite mechanisms of recognition and memory and timeline of action. This view has important implications for the understanding of immune mechanisms of memory. Innate recognition mechanisms are highly conserved across phyla, driving the specific adaptive immune responses in higher vertebrates (Sánchez-Ramón and Butnaru, 2013). Meanwhile, adaptive memory strategies correspond to evolutionarily innovated and biochemically distinct highly diverse specific receptors capable of generating a more rapid, efficient and sophisticated response through memory. Therefore, innate control of adaptive immunity is now a well-established paradigm (Iwasaki and Medzhitov, 2015), which warrants the regulation of a more complex level of immunity in terms of economy and avoidance of potentially harmful responses.
In contrast, the present-day classification of two major types of CNS memory in implicit and explicit stems from its psychological content-perceptual and motor skills and without awareness vs. conscious contextual and semantic; and from its anatomical involvement-non-medial temporal lobe (MTL)-mediated vs. MTL-mediated-(Scoville and Milner, 1957, 2000; Graf and Schacter, 1985; Squire, 1992). Thus, MTL (hippocampus and adjacent structures) has been to date ascribed to explicit memory, what we are going to challenge here. Implicit and explicit types of memory have been classically described as quite independent of each other (Graf and Schacter, 1985; Schacter, 1987), which are referred to as dissociation and show neuroimaging correlates (Korsnes and Magnussen, 2014).

By analogy with the innate control of adaptive IS, we postulate that MTL is part of the innate (implicit) system and exerts control of explicit memory. Indeed, MTL homolog structures exist across invertebrate taxa, such as the mushroom bodies of arthropods (Kandel and Abel, 1995; Wolff and Strausfeld, 2015). Many areas of complex behavior rely on brain systems that are separate but operate in cooperative fashion. Consequently, MTL may have a dual role as seat for implicit memories and in giving rise to explicit ones. By contrast, the neocortex is unique among higher vertebrates and has evolved independently in humans (Karten, 1991; Roth and Dicke, 2005, 2012; Wang et al., 2010; Bruner and Iriki, in press) and hence, we can conjecture that the neocortex might account for the development of explicit memory, as we are going to explain below in a more in-depth way (Box 2).

The pioneering seminal discovery of the protein substrate for implicit memory consolidation in the sea slug Aplysia californica corresponds to the cytoplasmic polyadenylation element binding protein or CPEB (Si et al., 2003a). CPEB is involved in the specificity and strengthening of the synapse, showing functional self-perpetuating prion-like properties ( $\mathrm{Si}$ et al., 2003b). This protein seems to be highly conserved through phyla for noxious memory traces in neural circuits in both invertebrates and vertebrates including the human brain (Kandel et al., 2014). Prion-like proteins have also been ascribed in explicit memory circuits (Barco et al., 2006), and have been suggested as common molecular mechanisms to both implicit and explicit memory types. However, we may hypothesize that distinct alternative evolutionary processes have developed from the proteins specialized in innate memory processes to the specific-content-discriminating memories in the vertebrates' brain. MTL is necessary but not sufficient for explicit memory consolidation, as MTL lesions have shown to abrogate newly acquired memories and learning but not previously acquired long-term consolidated memories (Scoville and Milner, 1957, 2000).

\footnotetext{
BOX 2 | Overlay of classification of recognition and memory from the IS to the CNS.

- Innate and adaptive immunity support different strategies and molecular solutions of recognition and memory acquired during evolution, which fit with differential quality and duration of the memory.

- Accordingly, we propose that implicit (perceptual and motor skills without awareness) and explicit (conscious contextual and semantic skills) memory should be explained by different strategies and molecular solutions.
} 
On the other hand, a recent revision of the 100-year-old brain anatomy classification by using gene-expression and embryology studies have enabled more accurate evolutionary brain models that has entirely changed its functional interpretation. In fact, an agreed amendment of the anatomical nomenclature of the avian brain has lead to a divergent analysis of much more complex cognitive abilities of some brain regions than previously appreciated. In particular, the nuclear avian pallium becomes to be considered homolog to the six-layered cortex or neocortex in mammals (Jarvis et al., 2005). The authors state the necessity of a re-evaluation of different neuroarchitectural solutions between avians and mammals to interpret sophisticated cognitive behaviors and to favor the emergence of new insights.

Altogether, we believe that the integrated model of innate and adaptive immune mechanisms may provide a conceptual framework for studying the roles of comparable neurobiological strategies of recognition, learning and memory.

\section{INTEGRATING INNATE AND ADAPTIVE RECOGNITION AND MEMORY STRATEGIES IN IMMUNE AND NERVOUS SYSTEMS}

\section{Innate or Implicit Strategies}

The IS and the CNS are extremely efficient systems along ancestral innate strategies from invertebrates to higher vertebrates. In the IS, the recognition strategies are either conserved throughout evolution or the result of convergent evolution in insects and vertebrates, as recently suggested for some toll-like receptors (TLRs; Hughes, 1999; Luo and Zheng, 2000; Kanzok et al., 2004). The traditional paradigm stresses the view that our innate IS encompasses mechanical barriers, secretions and soluble proteins at the front-line of host defense, as well as a large set of receptors that sense for exogenous and endogenous signals. Largely invariant shared microbial molecules are recognized by pathogen pattern recognition receptors (PRRs; Table 1). These interactions can induce danger signals and cellular stress, initiating the effector immune response (Gallucci and Matzinger, 2001; Janeway and Medzhitov, 2002; Hoffmann, 2003; Akira et al., 2006); or signals leading to the tolerogenic response (Cavaillon et al., 2003; Li et al., 2006). PRRs include TLRs, nucleotide-binding oligomerization domain (Nod)-, leucine-rich repeat-containing receptors (NLRs), RIG-Ilike receptors (RLRs), C-type lectin receptors (CLRs) and AIM-2 like receptors, as well as intracellular sensors of nucleic acids (Thery and Amigorena, 2001; Iwasaki and Medzhitov, 2015). Interestingly, these ligand-receptor interactions occur within a range of affinity and also multiple specificities, highlighting the biologic relevance of competitive and regulatory mechanisms (Miyake, 2007; Kondo et al., 2012). For instance, TLR4 can bind to bacterial lipopolysaccharides (LPS), but also to cellular hsp70 and hyaluronan (Matzinger, 2002). Despite some putative change of sensitivity status when second messenger accumulates (e.g., cyclic GMP-AMP synthase or cGAS products), no memory is ascribed to this line of strategies, which could be defined as intrinsic immunity (Table 1). Finally, these PRRs are also present in cells of the adaptive system, as a result of co-evolution.

A high degree of genetic degeneracy and redundancy (Edelman and Gally, 2001) together with acquired compensation mechanisms in the innate IS warrants survival. In fact, deficiencies of any PRRs result very rarely in life-threatening infections. Exceptions are the congenital TLR3-TRIF pathway deficiency associated with herpes simplex virus encephalitis (Casrouge et al., 2006); and the MyD88- and IL-1R associated kinase (IRAK)-4-dependent pathway that is essential for protective immunity to a few pyogenic bacteria in childhood but otherwise redundant in host defense (Casanova et al., 2011). An example of mechanisms of acquired compensation in this latter IRAK-4 deficiency displaying abolished antiviral TLR-7, -8 and -9 responses is illustrated by the maintenance of normal resistance to virus through TLR-3 and -4 (Yang et al., 2005).

Similarly, the CNS encompasses a large set of multiple sensor modalities with an extremely wide variety of receptors (visual, auditory, olfactory, gustative, kinaesthetic and somatosensory) that converge to primary and secondary sensory cortex and to the brainstem. These interactions could be operating in terms of threats or rewards conferred by external and internal environmental signals. Examples of convergent evolution within the CNS have been reported in invertebrates and vertebrates for olfactory and vision organs (Eisthen, 2002; Ogura et al., 2004; Gehring, 2014) or even in shared mechanisms of cell memory signaling for the IS and the CNS, such as NFkB (Friedman and Hughes, 2002; Lindsley et al., 2014). In analogy to the IS pattern, we could call it as innate intrinsic sensing strategy, giving rise to unconscious stereotyped or automated responses.

Synaptic neural plasticity exemplifies degeneracy in its full meaning (Edelman and Gally, 2001). A high degree of degeneracy, redundancy and dispersion in the implicit memory has been explained in terms of resonance in the network (Reber, 2013). For instance, genetic degeneracy under the lack of sensory receptors is apparent in subterranean mammals such as the blind mole rat, functionally blind and with poor auditory sensitivity but extremely efficient for spatial orientation thanks to a specialized self-generated echolocation system (Kimchi et al., 2004). As an example for acquired compensatory adjustments, the lack of specific sensory nervous receptors, as in human blindness, is associated with compensatory hypertrophy of other sensory modalities (Théoret et al., 2004) and with significant increase in the cerebellar areas coordinating sensory-motor interactions to compensate for the lack of visual information (Lepore et al., 2010).

\section{"Trained" Memory Strategies}

The traditional classification of two, i.e., innate and adaptive IS, from an evolutionary perspective has recently being challenged by the discovery of a transient pseudospecific memory within the innate IS, which represents a paradigm shift in immunity (Netea et al., 2015). Indeed, it is becoming increasingly acknowledged that cells of the innate IS can adapt following a previous encounter with a pathogen in what has been called "trained 
TABLE 1 | Proposed parallel classification of recognition and memory strategies.

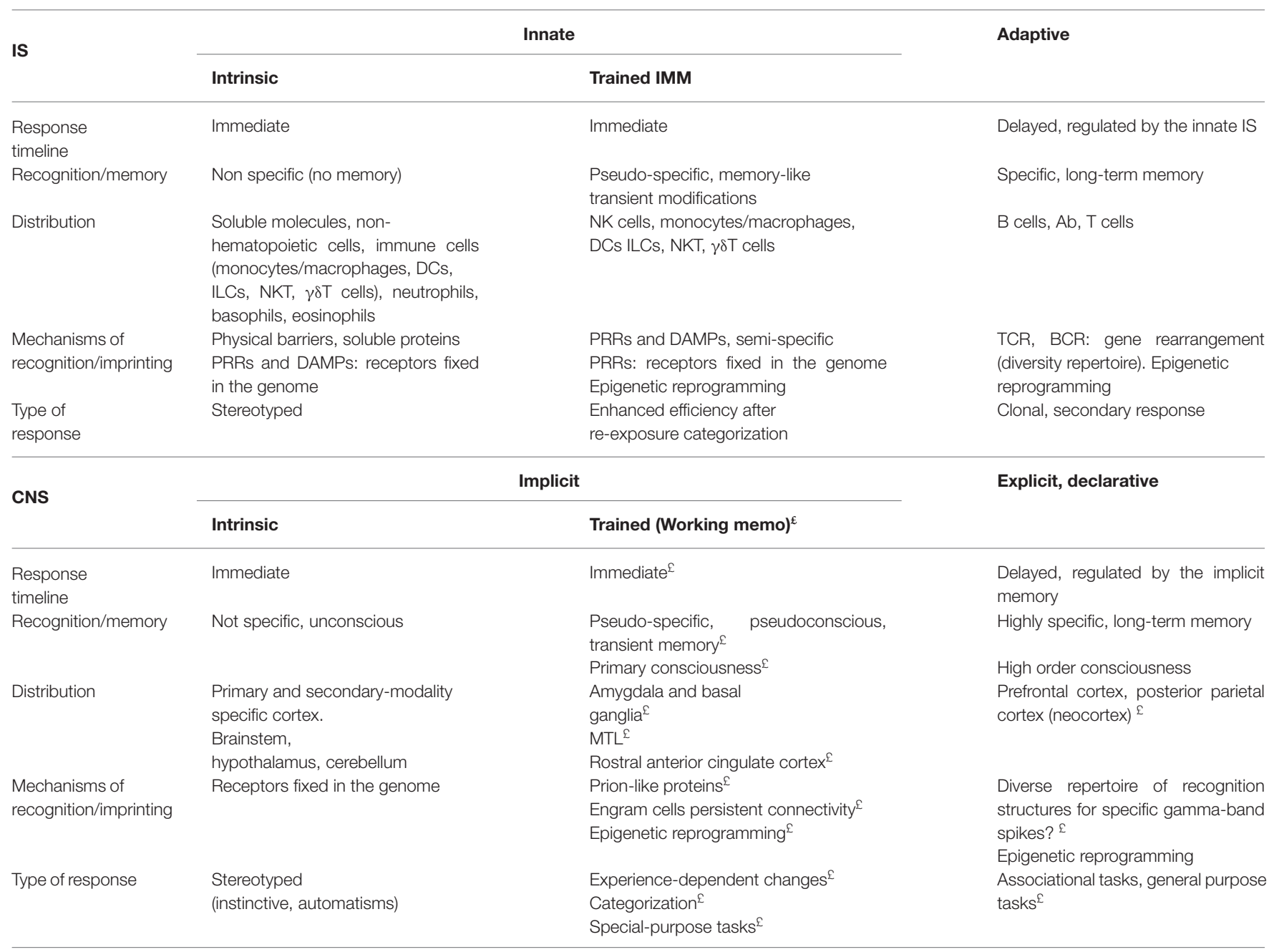

Ab, antibody; BCR, B cell receptor; DAMPs, Damage-associated molecular pattern molecules; DC, dendritic cells; ILCs, innate lymphoid cells; NKT, Natural Killer T cells; MTL, medial temporal lobe (hippocampus, entorhinal cortex, perirhinal, parahippocampal); PRR, pattern recognition receptors; TCR, $T$ cell receptor. ${ }^{E}$ Categories and features proposed in the model.

immunity" (Netea et al., 2011). Invertebrates have proven enhanced secondary immune responses in the absence of adaptive IS (Faulhaber and Karp, 1992) and the capability to transmit unspecific protection to their offspring against original and unrelated pathogens (Rheins et al., 1980). Mammalian prototypical innate immune cells such as NK cells (O'Leary et al., 2006; Sun et al., 2009) or monocytes (Quintin et al., 2012) elicit rapid responsiveness on second encounters, prolonging their survival and self renewal. This trained immunity is antigenpseudospecific and transient (Netea et al., 2015).

A similar capacity of such pseudospecific transient or "trained" memory may be observed in innate CNS structures, as can be inferred from the following facts (Box 3): (i) evidence for invertebrate brain memory for noxious cues within the model of Aplysia in the absence of explicit memory exists. The molecular mechanisms of this long-lasting synaptic plasticity in Aplysia and in the mammalian hippocampus (MTL) have proven to have many similarities (Pittenger and Kandel, 2003), and involve the regulation of prion-like protein synthesis at specific dendritic spines; (ii) Hippocampus-like structures called mushroom bodies are present in various invertebrates like bees, insects that exhibit cross-sensory integration, associative learning and memory storage and retrieval. This memory is necessary for the insect to locate multiple places in the environment, as well as for social life (Menzel, 2014); and (iii) Several empirical evidences support the involvement of MTL on implicit and unaware learning tasks besides its undeniable link to explicit memory (Hannula and Greene, 2012; Reber, 2013). Supporting this concept, the works of Dragoi and Tonegawa (2013) have unveiled how fear (innate) contextual cues activate selective ensembles or engrams of hippocampal neurons that contain pre-representations of the external world by specific temporal theta firing sequences in mice. In contrast, high gamma synchrony correlates with explicit (adaptive) awareness 


\begin{abstract}
BOX 3 | Facts further supporting MTL as innate CNS structure.
- Evidence for regulation of prion-like protein synthesis at specific dendritic spines after noxious cues in invertebrates and vertebrates (MTL).

- Mushroom bodies are hippocampus-like structures in invertebrates like bees that coordinate cross-sensory integration, associative learning and memory storage and retrieval for up to several weeks.

- Cumulative empirical evidence on the role of MTL in implicit as well as in explicit memory.
\end{abstract}

of spatial working memory (Yamamoto et al., 2014). Recall for fear contextual memories in mice is primarily based on the re-activation of the same engrams of hippocampal cells activated during initial encoding. This suggests that the memory would reside in these retained specific patterns of connectivity, whereas the strengthening of synapses would be driving the access to activation of such engrams, given that protein synthesis inhibitor did not abrogate engram activation (Ryan et al., 2015). These results are in line with the view that the hippocampus (MTL) is critically involved in implicit fearconditioned memories, and that its danger-fear valence may be manipulated by concomitant positive stimulus (Redondo et al., 2014). On the other hand, human functional neuroimaging studies have shown the activation of MTL immediately after termination of a new stimulus, referred as hippocampal offsetlocked response, predicting the registration of the episodic memories (Ben-Yakov and Dudai, 2011; Ben-Yakov et al., 2013).

\section{Control of Adaptive Memory by Innate Memory Strategies}

As previously stated, the initiation of the adaptive immune response depends on the triggering of innate receptors such as PRRs on the antigen-presenting cells (APCs). APCs such as dendritic cells (DC) are short lived cells specialized in the presentation of antigens to lymphocytes and in the priming the adaptive immune response (Figure 1). These PRRs categorize the origin of the antigens recognized by antigenspecific $\mathrm{T}$ and $\mathrm{B}$ cells and define the type of response. Consequently, lymphocytes are instructed to differentiate into the appropriate effector class of the immune response depending on the context of presentation (Iwasaki and Medzhitov, 2015). In this setting, specificity but also a certain degree of degeneracy for essentially any antigen can act as a big source of information that can be used freely to adopt enormous numbers of alternative responses. As an advocacy fact supporting this concept, defective signaling of particular TLRs in APCs have been ascribed to impaired recognition and memory responses in prototypical adaptive immune pathologies, namely in autoimmune diseases; and even in congenital immunodeficiencies, such as common variable immunodeficiency (Yu et al., 2009; Mills, 2011).

In our model, this archetypal APC-dependance referred to the human CNS implies the existence of structures a priori, namely the hippocampus and adjacent areas (MTL), which would act as the biological substrate of categorization, learning and first step for explicit memory (Figure 1 and Box 4). This a priori structure conditionally underlies to human reason in what Kant (1998) defined as the transcendental. It is through such categories that we conceive and think objects. Similarly to what has been described for the APCs, hippocampal cells are in constant renewal and show complex topographical and functional specialization.

\section{Adaptive or Explicit Strategies}

The ubiquity, diversity and rapid co-evolvability of pathogens and host require more complex mechanisms of response. A clonal exceedingly diverse anticipatory repertoire in which each lymphocyte bears a unique antigen-binding receptor-T cell receptors (TCRs) or $\mathrm{B}$ cell receptors (BCR or immunoglobulins)-is the central feature of the adaptive IS that evolved in the vertebrate ancestors (Cooper and Alder, 2006). The genes encoding the TCR and $\mathrm{BCR}$ are assembled from variable and constant fragments through recombination-activating genes (RAGs) protein-mediated somatic recombination, which yields an extensive variation in the repertoire of receptors (BCR with $10^{11}$ potential combinations and TCR with $10^{16}$ ). Mechanisms such as non-template nucleotide addition, gene conversion and somatic hypermutation for B cell further increase the diversity and specificity in adaptive immune recognition.

In parallel with what has been described for the IS, brain highly specific recognition refers to discrimination of what-isit type of data characterized by extreme precision and accuracy to the memory. This relies on selection process operating among the diverse repertoire of populations of neurons and on globally coordinated brain activation or consciousness. Current evolutionary theories on human cognitive processes highlight the relevance of the conjoint parieto-frontal neocortex system (Vakhtin et al., 2014) and the precuneus ("the eye of the self"; Freton et al., 2014; Bruner and Iriki, in press). Despite an extensive search for DNA rearrangement in the CNS, no locus has been identified to date. Given the highly precise topographical functional organization of brain cells, the actual location of a single neuron contributes to its virtually unique identity (Shatz, 1996; Chun, 2001). The neocortical neurons are immensely diverse $\left(2.1-2.6 \times 10^{10}\right.$; Pelvig et al., 2008). A single cortical neuron can, not only receive inputs from a single cell, but rather a huge number of synapses that has not been uniformly estimated, varying from 5000 to 10,000 (Tang et al., 2001) to up to 30,000 (Rockland, 2002), strikingly comparable ciphers to those described for Ig and TCR. How they are selected for a specific neural signal is still controversial and the structural components of the specific memory remain an enigma.

Consider the specialization of neurons in the visual cortex: feature detection yields a huge diversity of specific patterns that match their receptive fields (Moser et al., 2014). Visual stimuli recruit intrinsically generated cortical circuits of neurons (ensembles) during embryogenesis prior to visual experience as a result of spontaneous activity generated in the retina (Shatz, 1996; retinal and cortical waves). An alternative proposed process involves gap junctions coupling of clonally related neurons at prenatal developmental stage (Li et al., 2012). In both cases, 


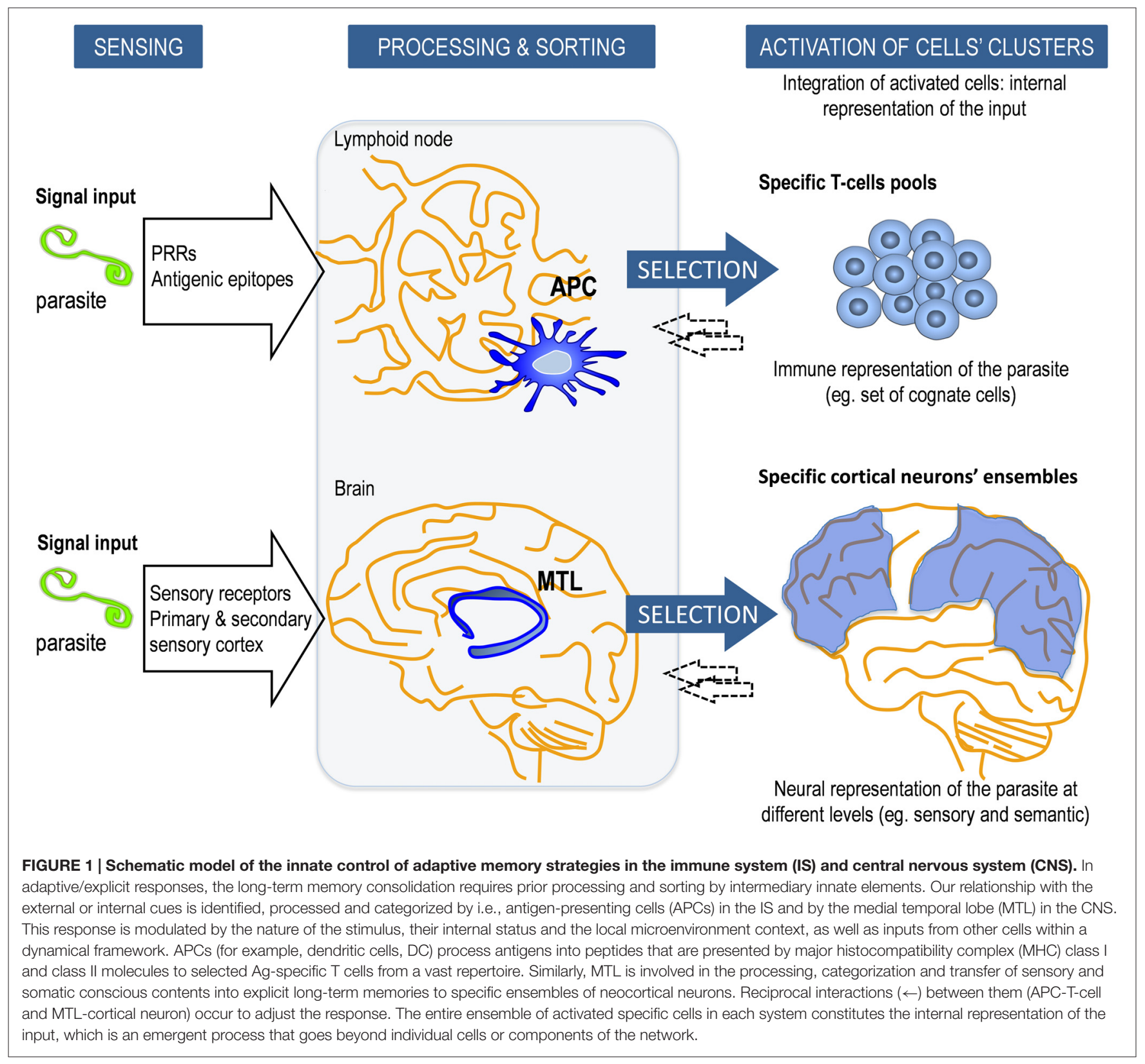

a selective competitive process requiring the formation and elimination of synaptic connections takes place (Shatz, 1996). Strikingly, patients with congenital blindness due to Leber's congenital amaurosis (LCA) who underwent gene therapy of retinal receptors showed a similar organization of visual circuitries and areas after first visual experience to those in healthy subjects (Ashtari et al., 2015).

According to this view, we can hypothesize that the singularity of the synapse in a neocortical neuron for a specific cue would reside in its specific receptivity for a given codified oscillatory signal sent from the MTL; as long as the singularity of the T-lymphocyte resides in its specific recognition of the peptide presented by the APC (Box 5). In this regard, gamma-band activity has been
BOX 4 | Role of the MTL: overlay with an immune system paradigm. In parallel with the paradigm of the innate control of adaptive immunity, here we present an immunological-based model for brain memory:

- APCs have the capability to form transient and pseudospecific memory upon encounter with the antigen (trained memory); and drive the specific $T$ and $B$ responses.

- MTL can integrate transient and pseudospecific memory and drive the formation of long-term explicit memory.

widely reported to be involved in the cognitive process in general (Varela et al., 2001). An alternative but not exclusive hypothesis advocates that very long-term memories are stored as the pattern of holes in the perineuronal net (Tsien, 2013). 
BOX 5 | Parallelism between complex processes of adaptive/ explicit memory in the IS and CNS.

- Selection processes operate in populations of specific cells of each adaptive system that globally and coordinately contain a dynamic representation of the stimulus.

- Adaptive systems rely on highly diverse anticipatory repertoires for specific recognition in spatiotemporal coordinated responses.

- Specific sensitivity to a given codified oscillatory signal from the MTL might provide the singularity of the synapse in a neocortical neuron for a cue.

Moreover, specific recognition is possible without intermediary elements. That is the case of Ig or BCR, capable of recognizing conformational epitopes of antigens. In the CNS, direct route bypassing MTL for explicit semantic memories has been described recently in anterior temporal cortex, a model for associative memory (Merhav et al., 2015). This pathway is used for vocabulary learning by children, and also by amnesic patients with severe MTL lesions (Merhav et al., 2015). Both alternative pathways represent a rapid and direct specific response.

\section{Emergent Processes in Complex Networks} Adaptive mechanisms generate emergent processes, characteristic of complex systems as a whole, which do not exist in each individual element. For instance, the dynamical behavior of the immune network display non-random patterns of local connectivity of the immune cells coupled with largescale functional organization. Connectivity at distant sites is accomplished by the migratory capacity of immune cells, in a coherent coordinated response. Similarly, connection patterns of the cerebral cortex typically consists of local and long-range pathways, which renders the system extremely efficient, in what has been called small-world structure (Watts and Strogatz, 1998; Achard et al., 2006).

We broach here how these diverse anticipatory repertoires are modeled by experience through epigenetic marks, evolutionarilyconserved processes that bridge between the IS and the CNS. Epigenetic marks are crucial to normal development (Fan et al., 2001), since these self-perpetuating modifications of gene expression are stably inherited by daughter cells after cell division, contributing to cellular identity despite constant molecular turnover (Turner, 2002). Epigenetic marks account also for the generation and stability of short-term (trained immunity) and long-term (adaptive immunity) memory of novel as well as of recall antigens (Youngblood et al., 2013; Saeed et al., 2014), evoking the concept of recontextualization of the memory. Epigenetic mechanisms operating in brain memory consolidation have been only recently proposed (Day and Sweatt, 2010). Diverse epigenetic mechanisms seem to be responsible of the permanence of synapse-specific changes, including enzyme-catalyzed histone modifications and noncoding RNAs (microRNAs, piwi-interacting RNAs), among others (Guan et al., 2002; Joilin et al., 2014).

\section{RECALL: A JOURNEY INTO THE PAST}

Retrieval or recall requires link of current cues to past history memory imprintings within a precise spatial cell interaction context. An ensemble of antigen-specific memory T lymphocytes' repertoire to different epitopes of a particular pathogen or tumor can be retained for a lifetime, implying an heterogeneous selection for multiple high-affinity T-lymphocyte pools that changes over time (Faure et al., 1998). The hallmark of adaptive IS memory is a faster and stronger response upon reencounter with a known antigen. APCs are critical intermediary elements also in recall responses. The site and timelapse of immune stimulation are key factors for obtaining the appropriate memory and effector responses (Huard et al., 1994) as exploited for immunotherapy design (Zitvogel and Faure, 1999). The exact mechanisms underlying time and locationdepending equations are still not well known. However, realtime visualization studies have revealed the differential behavior of antigen-experienced and naïve $\mathrm{T}$ lymphocytes following activation and the relevance of coordinated bidirectional communication with innate immune cells (Chtanova et al., 2009; Hor et al., 2015; Luu and Coombes, 2015).

In parallel, immune recall finds its echoes in brain recall mechanisms as a whole. Brain recall entails the activation of an extensive cortical brain network of specific neurons (Ryan et al., 2001; Cabeza et al., 2008). Again, the role of hippocampus in explicit recall is intriguing (Goshen et al., 2011). The hippocampus seems to be normally required for activation of neocortical memory traces depending on the quality of the memory. Reciprocal recursive and reverberating interactions are essential: the prefrontal cortex seems to facilitate top-down selection and organization of retrieval content (Shimamura, 2011), as well as updating relevant features; while the parietal cortex interacts with the MTL and the prefrontal cortex (Cabeza et al., 2008). Aspects of the same memory cut across all levels of hierarchy (semantic, lexical, phonetical, image), each level holding distinct degrees of detail through different time spans. This also evokes the relational nature of memory, not only of individual items but also of compositions of them in associative memory.

Brain lesion magnitude and temporal precision are crucial for remote recall compensation, whereas recent memory relies on hippocampal memory traces (Goshen et al., 2011).

\section{CONCLUSIONS}

The methodological standpoint used here enables to approach as a whole the large-scale of CNS network complexity and those emergent processes arising from interactions among multiple neurons and neural circuits. Besides, bringing together the different biological, psychological, clinical and evolutionary data opens a holistic dimension of CNS memory. This approach might help to overcome the inherent difficulty of such a complex system like the human brain being studied by itself. Still, mind transcends any reductionist view of the brain system.

Theoretical models are important tools for many aspects of scientific activity. Our model challenges the traditional classification of brain memory based on a psychological and anatomical perspective. It could reconcile the previous concepts with that of evolutionary and functionally equivalent homologies. This immune-based classification could provide 
new insights to the established psychological concepts inferred from clinical and behavioral data. The model emphasizes the need of a more accurate categorization of the quality of memories according to the different mechanisms and in an evolutionary context. A major advance has been achieved on the nature and mechanisms underlying innate memories (for fear-contextual memories), which account for specific and persistent engrams cells connectivity at the MTL (Ryan et al., 2015). Our present model supports the view that MTL, as APC for the IS, represents part of the innate memory strategies and a necessary step to adaptive or explicit memory type, instead of what has been to date the mainstream of neuroscientific thought (Scoville and Milner, 1957; Eichenbaum et al., 2007). The proposed classification leaves the neocortex as an evolutionarily acquired highly specific and permanent memory with an open question about the essence of its substrate. In line with our hypothesis, recent findings indicate divergent structure and turnover regulation in hippocampal and neocortical synapses that match with their temporal patterns of memory (transient vs. stable, respectively), suggesting differentially specialized structures in both systems (Attardo et al., 2015). In this study, glutamate receptor blockade contrarily impacted hippocampal and neocortical memory connectivity (Attardo et al., 2015).

In contrast to the accepted dissociation of implicit and explicit memory types by which MTL makes part of the explicit system (Graf and Schacter, 1985; Schacter, 1987), our current model defines however that explicit memory is tightly regulated by the implicit (MTL) system. We explain here this regulation not as a constraint imposed by the system but rather as an evolutionary-emerged property, further modulated in the setting of a historical process (referred to the interplay of genetic, epigenetic and developmental influences). Categories of adaptive responses are predefined by these intermediary elements (MTL and APC) prior to the relation with the object, acquired during embryogenesis and modified by experience all along lifetime. This is compatible with the Kantian concept of human reason, which postulates that our brain does not reflect the world outside but instead it is the world that reflects our brain. For example, the flavor of the chocolate is not in the chocolate itself but in its interactions with our taste buds and sensory areas and the way we perceive it. The same could be inferred for our IS, the immune world is the representation of the interactions of antigens with the immune architecture. Upon this tightly regulated architecture, experience-dependent plasticity through indelible epigenetic mechanisms take place, Further on, as intuited by Heidegger (1962) when analyzing the fundamentals of our reason and his reading on Kant, human understanding acquires its full sense within the dimension of

\section{REFERENCES}

Achard, S., Salvador, R., Whitcher, B., Suckling, J., and Bullmore, E. (2006). A resilient, low-frequency, small-world human brain functional network with highly connected association cortical hubs. J. Neurosci. 26, 63-72. doi: 10. 1523/jneurosci.3874-05.2006

Akira, S., Uematsu, S., and Takeuchi, O. (2006). Pathogen recognition and innate immunity. Cell 124, 783-801. doi: 10.1016/j.cell.2006.02.015 temporality. Altogether, epigenetics could be comprehended as the environmental signature on the individual uniqueness or, in philosophical terms, the source of improvisation (Malabou, 2014).

In both evolutionary acquired adaptive systems, experience is faced with exceedingly diverse pre-formed anticipatory elements for specific recognition in form of patterned associative responses, which in turn establish memory encoding and retrieval in both systems. Spatial temporal precision of interaction of cells ensembles within the network is essential in determining the response and requires bidirectional communications between innate/implicit and adaptive/explicit systems. Not paradoxically, artificial cognitive systems have been built as a powerful metaphor of the natural CNS and IS principles (pattern recognizer, classifier systems based, dynamic learning, error tolerance, anomaly and intrusion detection algorithms, robustness; Jacobsen et al., 2011; Fernandez-Leon et al., 2014).

\section{CONCLUDING REMARKS}

The neuron doctrine deals with the first order of approximation to the functioning of the brain that neglects associative patterns and emergent processes of the high order of neural network. By applying analogical reasoning between relationships of the IS and the CNS, we can hold the frame of high order interactions within an evolutionary context. This model leads to the concept that MTL integrates the innate or implicit memory system, giving rise to pseudo-specific memories, and controls the more complex and evolutionary recent neocortex, able of highly specific or explicit memories. We believe that this immune-based model and the new concepts derived from it could inspire the advent of new conceptualizations of large-scale brain networks and empirical approaches towards a unified theory of brain memory.

\section{AUTHOR CONTRIBUTIONS}

SS-R and FF have equally contributed to the conception and design of the manuscript, drafting, critical revision and final approval of the article. SS-R and FF agree to be accountable for all aspects of the work in ensuring that questions related to the accuracy or integrity of any part of the work are appropriately investigated and resolved.

\section{ACKNOWLEDGMENTS}

We thank Juan Álvarez-Linera, Ángel Corbí and Pablo J Sáez for their valuable comments that helped to shape the overall form of the article.

Ashtari, M., Zhang, H., Cook, P. A., Cyckowski, L. L., Shindler, K. S., Marshall, K. A., et al. (2015). Plasticity of the human visual system after retinal gene therapy in patients with Leber's congenital amaurosis. Sci. Transl. Med. 7:296ra110. doi: 10.1126/scitranslmed. aaa8791

Attardo, A., Fitzgerald, J. E., and Schnitzer, M. J. (2015). Impermanence of dendritic spines in live adult CA1 hippocampus. Nature 523, 592-596. doi: 10. 1038 /nature14467 
Barco, A., Bailey, C. H., and Kandel, E. R. (2006). Common molecular mechanisms in explicit and implicit memory. J. Neurochem. 97, 1520-1533. doi: 10.1111/j. 1471-4159.2006.03870.x

Barreiro, L. B., and Quintana-Murci, L. (2010). From evolutionary genetics to human immunology: how selection shapes host defence genes. Nat. Rev. Genet. 11, 17-30. doi: $10.1038 / \operatorname{nrg} 2698$

Barry, G., and Mattick, J. S. (2012). The role of regulatory RNA in cognitive evolution. Trends Cogn. Sci. 16, 497-503. doi: 10.1016/j.tics.2012.08.007

Ben-Yakov, A., and Dudai, Y. (2011). Constructing realistic engrams: poststimulus activity of hippocampus and dorsal striatum predicts subsequent episodic memory. J. Neurosci. 31, 9032-9042. doi: 10.1523/JNEUROSCI.0702-11.2011

Ben-Yakov, A., Eshel, N., and Dudai, Y. (2013). Hippocampal immediate poststimulus activity in the encoding of consecutive naturalistic episodes. J. Exp. Psychol. Gen. 142, 1255-1263. doi: 10.1037/a0033558

Bruner, E., and Iriki, A. (in press). Extending mind, visuospatial integration and the evolution of the parietal lobes in the human genus. Quat. Int. 1-13. doi: 10. 1016/j.quaint.2015.05.019

Cabeza, R., Ciaramelli, E., Olson, I. R., and Moscovitch, M. (2008). The parietal cortex and episodic memory: an attentional account. Nat. Rev. Neurosci. 9, 613-625. doi: 10.1038/nrn2459

Casanova, J. L., Abel, L., and Quintana-Murci, L. (2011). Human TLRs and IL-1Rs in host defense: natural insights from evolutionary, epidemiological and clinical genetics. Annu. Rev. Immunol. 29, 447-491. doi: 10.1146/annurev-immunol030409-101335

Casrouge, A., Zhang, S. Y., Eidenschenk, C., Jouanguy, E., Puel, A., Yang, K., et al. (2006). Herpes simplex virus encephalitis in human UNC-93B deficiency. Science 314, 308-312. doi: 10.1126/science. 1128346

Cavaillon, J. M., Adrie, C., Fitting, C., and Adib-Conquy, M. (2003). Endotoxin tolerance: is there a clinical relevance? J. Endotoxin Res. 9, 101-107. doi: 10. $1177 / 09680519030090020501$

Chtanova, T., Han, S. J., Schaeffer, M., van Dooren, G. G., Herzmark, P., Striepen, B., et al. (2009). Dynamics of T cell, antigen-presenting cell and pathogen interactions during recall responses in the lymph node. Immunity 31 , 342-355. doi: 10.1016/j.immuni.2009.06.023

Chun, J. (2001). Selected comparison of immune and nervous system development. Adv. Immunol. 77, 297-322. doi: 10.1016/s0065-2776(01) 77020-4

Cooper, M. D., and Alder, M. N. (2006). The evolution of adaptive immune systems. Cell 124, 815-822. doi: 10.1016/j.cell.2006.02.001

Day, J. J., and Sweatt, J. D. (2010). DNA methylation and memory formation. Nat. Neurosci. 13, 1319-1323. doi: 10.1038/nn.2666

Dorus, S., Vallender, E. J., Evans, P. D., Anderson, J. R., Gilbert, S. L., Mahowald, M., et al. (2004). Accelerated evolution of nervous system genes in the origin of Homo sapiens. Cell 119, 1027-1040. doi: 10.1016/j.cell.2004.11.040

Dragoi, G., and Tonegawa, S. (2013). Distinct preplay of multiple novel spatial experiences in the rat. Proc. Natl. Acad. Sci. U S A 110, 9100-9105. doi: 10. 1073/pnas. 1306031110

Edelman, G. M., and Gally, J. A. (2001). Degeneracy and complexity in biological systems. Proc. Natl. Acad. Sci. U S A 98, 13763-13768. doi: 10.1073/pnas. 231499798

Eichenbaum, H., Yonelinas, A. P., and Ranganath, C. (2007). The medial temporal lobe and recognition memory. Annu. Rev. Neurosci. 30, 123-152. doi: 10. 1146/annurev.neuro.30.051606.094328

Eisthen, H. L. (2002). Why are olfactory systems of different animals so similar? Brain Behav. Evol. 59, 273-293. doi: 10.1159/0000 63564

Fan, G., Beard, C., Chen, R. Z., Csankovszki, G., Sun, Y., Siniaia, M., et al. (2001). DNA hypomethylation perturbs the function and survival of CNS neurons in postnatal animals. J. Neurosci. 21, 788-797.

Faulhaber, L. M., and Karp, R. D. (1992). A diphasic immune response against bacteria in the American cockroach. Immunology 75, 378-381.

Faure, F., Even, J., and Kourilsky, P. (1998). Tumor-specific immune response: current in vitro analyses may not reflect the in vivo immune status. Crit. Rev. Immunol. 18, 77-86. doi: 10.1615/critrevimmunol.v18.i1-2.90

Fernandez-Leon, J. A., Acosta, G. G., and Rozenfeld, A. (2014). How simple autonomous decisions evolve into robust behaviours? A review from neurorobotics, cognitive, self-organized and artificial immune systems fields. Biosystems 124, 7-20. doi: 10.1016/j.biosystems.2014.08.003
Freton, M., Lemogne, C., Bergouignan, L., Delaveau, P., Lehéricy, S., and Fossati, P. (2014). The eye of the self: precuneus volume and visual perspective during autobiographical memory retrieval. Brain Struct. Funct. 219, 959-968. doi: 10. 1007/s00429-013-0546-2

Friedman, R., and Hughes, A. L. (2002). Molecular evolution of the NF-kappaB signaling system. Immunogenetics 53, 964-974. doi: 10.1007/s00251-0010399-3

Gallucci, S., and Matzinger, P. (2001). Danger signals: SOS to the immune system. Curr. Opin. Immunol. 13, 114-119. doi: 10.1016/s0952-7915(00)00191-6

Gehring, W. J. (2014). The evolution of vision. Wiley Interdiscip. Rev. Dev. Biol. 3 , 1-40. doi: $10.1002 /$ wdev.96

Goshen, I., Brodsky, M., Prakash, R., Wallace, J., Gradinaru, V., Ramakrishnan, C., et al. (2011). Dynamics of retrieval strategies for remote memories. Cell 147, 678-689. doi: 10.1016/j.cell.2011.09.033

Graf, P., and Schacter, D. (1985). Implicit and explicit memory for new associations in normal and amnesic subjects. J. Exp. Psychol. Learn. Mem. Cogn. 11, 501-518. doi: 10.1037/0278-7393.11.3.501

Guan, Z., Giustetto, M., Lomvardas, S., Kim, J. H., Miniaci, M. C., Schwartz, J. H., et al. (2002). Integration of long-term-memory-related synaptic plasticity involves bidirectional regulation of gene expression and chromatin structure. Cell 111, 483-493. doi: 10.1016/s0092-8674(02)01074-7

Hannula, D. E., and Greene, A. J. (2012). The hippocampus reevaluated in unconscious learning and memory: at a tipping point? Front. Hum. Neurosci. 6:80. doi: 10.3389/fnhum.2012.00080

Hebb, D. O. (1949). Organization of Behaviour: A Neuropsychological Theory. New York: John Wiley and Sons.

Heidegger, M. (1962). Being and Time. NYC, Evanston: Harper and Row.

Henke, K. (2010). A model for memory systems based on processing modes rather than consciousness. Nat. Rev. Neurosci. 11, 523-532. doi: 10.1038/nrn2850

Hoffmann, J. A. (2003). The immune response of Drosophila. Nature 426, 33-38. doi: 10.1038/nature02021

Hor, J. L., Whitney, P. G., Zaid, A., Brooks, A. G., Heath, W. R., and Mueller, S. N. (2015). Spatiotemporally distinct interactions with dendritic cell subsets facilitates CD4+ and CD8+ T cell activation to localized viral infection. Immunity 43, 554-565. doi: 10.1016/j.immuni.2015.07.020

Huard, B., Tournier, M., Hercend, T., Triebel, F., and Faure, F. (1994). Lymphocyte-activation gene 3/major histocompatibility complex class II interaction modulates the antigenic response of CD4+ T lymphocytes. Eur. $J$. Immunol. 24, 3216-3221. doi: 10.1002/eji.1830241246

Hughes, A. L. (1999). Phylogenies of developmentally important proteins do not support the hypothesis of two rounds of genome duplication early in vertebrate history. J. Mol. Evol. 48, 565-576. doi: 10.1007/pl00006499

Iwasaki, A., and Medzhitov, R. (2015). Control of adaptive immunity by the innate immune system. Nat. Immunol. 16, 343-353. doi: 10.1038/ni.3123

Jacobsen, R. H., Zhang, Q., and Toftegaard, T. S. (2011). Bioinspired principles for large-scale networked sensor systems: an overview. Sensors (Basel) 11, 4137-4151. doi: 10.3390/s110404137

Janeway, C. A. Jr. and Medzhitov, R. (2002). Innate immune recognition. Annu. Rev. Immunol. 20, 197-216. doi: 10.1146/annurev.immunol.20.083001.084359

Jarvis, E. D., Gunturkun, O., Bruce, L., Csillag, A., Karten, H., Kuenzel, W., et al. (2005). Avian brains and a new understanding of vertebrate brain evolution. Nat. Rev. Neurosci. 6, 151-159. doi: 10.1038/ nrn1606

Joilin, G., Guévremont, D., Ryan, B., Claudianos, C., Cristino, A. S., Abraham, W. C., et al. (2014). Rapid regulation of microRNA following induction of longterm potentiation in vivo. Front. Mol. Neurosci. 7:98. doi: 10.3389/fnmol.2014. 00098

Kandel, E., and Abel, T. (1995). Neuropeptides, adenylyl cyclase and memory storage. Science 268, 825-826. doi: 10.1126/science.7754367

Kandel, E. R., Dudai, Y., and Mayford, M. R. (2014). The molecular and systems biology of memory. Cell 157, 163-186. doi: 10.1016/j.cell.2014.03.001

Kant, I. (1998). Critique of Pure Reason. Cambridge: Cambridge University press.

Kanzok, S. M., Hoa, N. T., Bonizzoni, M., Luna, C., Huang, Y., Malacrida, A. R., et al. (2004). Origin of Toll-like receptor-mediated innate immunity. J. Mol. Evol. 58, 442-448. doi: 10.1007/s00239-0032565-8

Karten, H. J. (1991). Homology and evolutionary origins of the 'neocortex'. Brain Behav. Evol. 38, 264-272. doi: 10.1159/000114393 
Kimchi, T., Etienne, A. S., and Terkel, J. (2004). A subterranean mammal uses the magnetic compass for path integration. Proc. Natl. Acad. Sci. U S A 101, 1105-1109. doi: 10.1073/pnas.0307560100

Kondo, T., Kawai, T., and Akira, S. (2012). Dissecting negative regulation of Tolllike receptor signaling. Trends Immunol. 33, 449-458. doi: 10.1016/j.it.2012. 05.002

Korsnes, M. S., and Magnussen, S. J. (2014). fMRI evidence for dissociation between priming and conscious recognition. J. Integr. Neurosci. 13, 509-517. doi: $10.1142 / \mathrm{s} 0219635214500149$

Lepore, N., Voss, P., Lepore, F., Chou, Y. Y., Fortin, M., Gougoux, F., et al. (2010). Brain structure changes visualized in early- and late-onset blind subjects. Neuroimage 49, 134-140. doi: 10.1016/j.neuroimage.2009.07.048

Li, Y., Lu, H., Cheng, P. L., Ge, S., Xu, H., Shi, S. H., et al. (2012). Clonally related visual cortical neurons show similar stimulus feature selectivity. Nature 486, 118-121. doi: 10.1038/nature11110

Li, C. H., Wang, J. H., and Redmond, H. P. (2006). Bacterial lipoprotein-induced self-tolerance and cross-tolerance to LPS are associated with reduced IRAK-1 expression and MyD88-IRAK complex formation. J. Leukoc. Biol. 79, 867-875. doi: 10.1189/jlb.0905505

Lindsley, A. W., Qian, Y., Valencia, C. A., Shah, K., Zhang, K., and Assa'ad, A. (2014). Combined immune deficiency in a patient with a novel NFKB2 mutation. J. Clin. Immunol. 34, 910-915. doi: 10.1007/s10875-014-0095-3

Luo, C., and Zheng, L. (2000). Independent evolution of Toll and related genes in insects and mammals. Immunogenetics 51, 92-98. doi: 10.1007/s002510050017

Luu, L., and Coombes, J. L. (2015). Dynamic two-photon imaging of the immune response to Toxoplasma gondii infection. Parasite Immunol. 37, 118-126. doi: $10.1111 /$ pim. 12161

Malabou, C. (2014). Avant Demain. ÉPigenèse Et Rationalité. Paris: Presses Universitaires de France.

Matzinger, P. (2002). The danger model: a renewed sense of self. Science 296, 301-305. doi: 10.1126/science.1071059

Medzhitov, R., and Janeway, C. A. Jr. (2002). Decoding the patterns of self and nonself by the innate immune system. Science 296, 298-300. doi: 10 . $1126 /$ science. 1068883

Menzel, R. (2014). The insect mushroom body, an experience-dependent recoding device. J. Physiol. Paris 108, 84-95. doi: 10.1016/j.jphysparis.2014.07.004

Merhav, M., Karni, A., and Gilboa, A. (2015). Not all declarative memories are created equal: fast mapping as a direct route to cortical declarative representations. Neuroimage 117, 80-92. doi: 10.1016/j.neuroimage.2015. 05.027

Mills, K. H. (2011). TLR-dependent T cell activation in autoimmunity. Nat. Rev. Immunol. 11, 807-822. doi: 10.1038/nri3095

Miyake, K. (2007). Innate immune sensing of pathogens and danger signals by cell surface Toll-like receptors. Semin. Immunol. 19, 3-10. doi: 10.1016/j.smim. 2006.12.002

Moser, E. I., Roudi, Y., Witter, M. P., Kentros, C., Bonhoeffer, T., and Moser, M. B. (2014). Grid cells and cortical representation. Nat. Rev. Neurosci. 15, 466-481. doi: $10.1038 / \mathrm{nrn} 3766$

Nabavi, S., Fox, R., Proulx, C. D., Lin, J. Y., Tsien, R. Y., and Malinow, R. (2014). Engineering a memory with LTD and LTP. Nature 511, 348-352. doi: 10. 1038/nature13294

Netea, M. G., Latz, E., Mills, K. H., and O’Neill, L. A. (2015). Innate immune memory: a paradigm shift in understanding host defense. Nat. Immunol. 16, 675-679. doi: 10.1038/ni.3178

Netea, M. G., Quintin, J., and van der Meer, J. W. (2011). Trained immunity: a memory for innate host defense. Cell Host Microbe 9, 355-361. doi: 10.1016/j. chom.2011.04.006

Ogura, A., Ikeo, K., and Gojobori, T. (2004). Comparative analysis of gene expression for convergent evolution of camera eye between octopus and human. Genome Res. 14, 1555-1561. doi: 10.1101/gr.2268104

O'Leary, J. G., Goodarzi, M., Drayton, D. L., and von Andrian, U. H. (2006). T celland B cell-independent adaptive immunity mediated by natural killer cells. Nat. Immunol. 7, 507-516. doi: 10.1038/ni1332

Pelvig, D. P., Pakkenberg, H., Stark, A. K., and Pakkenberg, B. (2008). Neocortical glial cell numbers in human brains. Neurobiol. Aging 29, 1754-1762. doi: 10. 1016/j.neurobiolaging.2007.04.013

Pittenger, C., and Kandel, E. R. (2003). In search of general mechanisms for longlasting plasticity: aplysia and the hippocampus. Philos. Trans. R. Soc. Lond. B Biol. Sci. 358, 757-763. doi: 10.1098/rstb.2002.1247
Quintin, J., Saeed, S., Martens, J. H., Giamarellos-Bourboulis, E. J., Ifrim, D. C., Logie, C., et al. (2012). Candida albicans infection affords protection against reinfection via functional reprogramming of monocytes. Cell Host Microbe 12, 223-232. doi: 10.1016/j.chom.2012.06.006

Ramón y Cajal, S. (1891). Sur la structure de l'ecorce cerebrale de quelques mamiferes. La Cellule 7, 124-176.

Reber, P. J. (2013). The neural basis of implicit learning and memory: a review of neuropsychological and neuroimaging research. Neuropsychologia 51, 2026-2042. doi: 10.1016/j.neuropsychologia.2013. 06.019

Redondo, R. L., Kim, J., Arons, A. L., Ramirez, S., Liu, X., and Tonegawa, S. (2014). Bidirectional switch of the valence associated with a hippocampal contextual memory engram. Nature 513, 426-430. doi: 10.1038/nature 13725

Rheins, L. A., Karp, R. D., and Butz, A. (1980). Induction of specific humoral immunity to soluble proteins in the American cockroach (Periplaneta americana). I. Nature of the primary response. Dev. Comp. Immunol. 4, 447-458. doi: 10.1016/s0145-305x(80)80047-4

Rockland, K. S. (2002). Non-uniformity of extrinsic connections and columnar organization. J. Neurocytol. 31, 247-253. doi: 10.1023/A:1024169925377

Roth, G., and Dicke, U. (2005). Evolution of the brain and intelligence. Trends Cogn. Sci. 9, 250-257. doi: 10.1016/j.tics.2005.03.005

Roth, G., and Dicke, U. (2012). Evolution of the brain and intelligence in primates. Prog. Brain Res. 195, 413-430. doi: 10.1016/B978-0-444-53860-4.00020-9

Ryan, L., Nadel, L., Keil, K., Putnam, K., Schnyer, D., Trouard, T., et al. (2001). Hippocampal complex and retrieval of recent and very remote autobiographical memories: evidence from functional magnetic resonance imaging in neurologically intact people. Hippocampus 11, 707-714. doi: 10. 1002/hipo.1086

Ryan, T. J., Roy, D. S., Pignatelli, M., Arons, A., and Tonegawa, S. (2015). Memory. Engram cells retain memory under retrograde amnesia. Science 348, 1007-1013. doi: 10.1126/science.aaa5542

Saeed, S., Quintin, J., Kerstens, H. H., Rao, N. A., Aghajanirefah, A., Matarese, F., et al. (2014). Epigenetic programming of monocyte-to-macrophage differentiation and trained innate immunity. Science 345:1251086. doi: 10. 1126/science. 1251086

Sánchez-Ramón, S., and Butnaru, D. (2013). "If immunity or tolerance was the response, what was the question? Reflection on immune recognition," in Medicina Evolucionista: Aportaciones Pluridisciplinares (Vol. 2), eds A. Daschner, J. L. Gómez Pérez, and M. J. Trujillo Tiebas (Madrid: MedEvo), $35-50$.

Schacter, D. L. (1987). Implicit expressions of memory in organic amnesia: learning of new facts and associations. Hum. Neurobiol. 6, 107-118.

Scoville, W. B., and Milner, B. (1957). Loss of recent memory after bilateral hippocampal lesions. J. Neurol. Neurosurg. Psychiatry 20, 11-21. doi: 10. 1136/jnnp.20.1.11

Scoville, W. B., and Milner, B. (2000). Loss of recent memory after bilateral hippocampal lesions. 1957. J. Neuropsychiatry Clin. Neurosci. 12, 103-113. doi: $10.1176 /$ jnp.12.1.103-a

Shatz, C. J. (1996). Emergence of order in visual system development. Proc. Natl. Acad. Sci. U S A 93, 602-608. doi: 10.1073/pnas.93.2.602

Shimamura, A. P. (2011). Episodic retrieval and the cortical binding of relational activity. Cogn. Affect. Behav. Neurosci. 11, 277-291. doi: 10.3758/s13415-0110031-4

Si, K., Giustetto, M., Etkin, A., Hsu, R., Janisiewicz, A. M., Miniaci, M. C., et al. (2003a). A neuronal isoform of CPEB regulates local protein synthesis and stabilizes synapse-specific long-term facilitation in aplysia. Cell 115, 893-904. doi: 10.1016/s0092-8674(03)01021-3

Si, K., Lindquist, S., and Kandel, E. R. (2003b). A neuronal isoform of the aplysia CPEB has prion-like properties. Cell 115, 879-891. doi: 10.1016/s00928674(03)01020-1

Squire, L. R. (1992). Declarative and nondeclarative memory: multiple brain systems supporting learning and memory. J. Cogn. Neurosci. 4, 232-243. doi: 10.1162/jocn.1992.4.3.232

Sun, J. C., Belke, J. N., and Lanier, L. L. (2009). Adaptive immune features of natural killer cells. Nature 457, 557-561. doi: 10.1038/nature07665

Tang, Y., Nyengaard, J. R., De Groot, D. M., and Gundersen, H. J. (2001). Total regional and global number of synapses in the human brain neocortex. Synapse 41, 258-273. doi: 10.1002/syn.1083 
Théoret, H., Merabet, L., and Pascual-Leone, A. (2004). Behavioral and neuroplastic changes in the blind: evidence for functionally relevant crossmodal interactions. J. Physiol. Paris 98, 221-233. doi: 10.1016/j.jphysparis.2004. 03.009

Thery, C., and Amigorena, S. (2001). The cell biology of antigen presentation in dendritic cells. Curr. Opin. Immunol. 13, 45-51. doi: 10.1016/s09527915(00)00180-1

Tsien, R. Y. (2013). Very long-term memories may be stored in the pattern of holes in the perineuronal net. Proc. Natl. Acad. Sci. U S A 110, 12456-12461. doi: 10. 1073/pnas.1310158110

Turner, B. M. (2002). Cellular memory and the histone code. Cell 111, 285-291. doi: 10.1016/s0092-8674(02)01080-2

Vakhtin, A. A., Ryman, S. G., Flores, R. A., and Jung, R. E. (2014). Functional brain networks contributing to the parieto-frontal integration theory of intelligence. Neuroimage 103, 349-354. doi: 10.1016/j.neuroimage.2014. 09.055

Varela, F. J., and Coutinho, A. (1989). Immune networks: getting on to the real thing. Res. Immunol. 140, 837-845. doi: 10.1016/0923-2494(89) 90043-6

Varela, F. J., and Coutinho, A. (1991). Second generation immune networks. Immunol. Today 12, 159-166. doi: 10.1016/s0167-5699(05)80046-5

Varela, F., Lachaux, J. P., Rodriguez, E., and Martinerie, J. (2001). The brainweb: phase synchronization and large-scale integration. Nat. Rev. Neurosci. 2, 229-239. doi: 10.1038/35067550

Wang, Y., Brzozowska-Prechtl, A., and Karten, H. J. (2010). Laminar and columnar auditory cortex in avian brain. Proc. Natl. Acad. Sci. U S A 107, 12676-12681. doi: 10.1073/pnas.1006645107

Watts, D. J., and Strogatz, S. H. (1998). Collective dynamics of 'small-world' networks. Nature 393, 440-442. doi: 10.1038/30918

Willingham, D. B., and Goedert, K. (2001). The role of taxonomies in the study of human memory. Cogn. Affect. Behav. Neurosci. 1, 250-265. doi: 10.3758/cabn. 1.3 .250
Wolff, G. H., and Strausfeld, N. J. (2015). Genealogical correspondence of mushroom bodies across invertebrate phyla. Curr. Biol. 25, 38-44. doi: 10. 1016/j.cub.2014.10.049

Yamamoto, J., Suh, J., Takeuchi, D., and Tonegawa, S. (2014). Successful execution of working memory linked to synchronized high-frequency gamma oscillations. Cell 157, 845-857. doi: 10.1016/j.cell.2014.04.009

Yang, K., Puel, A., Zhang, S., Eidenschenk, C., Ku, C. L., Casrouge, A., et al. (2005). Human TLR-7-, -8- and -9-mediated induction of IFN-alpha/beta and -lambda Is IRAK-4 dependent and redundant for protective immunity to viruses. Immunity 23, 465-478. doi: 10.1016/j.immuni.2005.09.016

Youngblood, B., Hale, J. S., and Ahmed, R. (2013). T-cell memory differentiation: insights from transcriptional signatures and epigenetics. Immunology 139, 277-284. doi: 10.1111/imm.12074

Yu, J. E., Knight, A. K., Radigan, L., Marron, T. U., Zhang, L., SánchezRamón, S., et al. (2009). Toll-like receptor 7 and 9 defects in common variable immunodeficiency. J. Allergy Clin. Immunol. 124, 349-356, 356.e1-343.e3. doi: 10.1016/j.jaci.2009.05.019

Yuste, R. (2015). From the neuron doctrine to neural networks. Nat. Rev. Neurosci. 16, 487-497. doi: 10.1038/nrn3962

Zitvogel, L., and Faure, F. (1999). L'immunité antitumorale: des concepts à l'immunothérapie active spécifique. Med. Sci. 8-9, 939-949.

Conflict of Interest Statement: The authors declare that the research was conducted in the absence of any commercial or financial relationships that could be construed as a potential conflict of interest.

Copyright (c) 2016 Sánchez-Ramón and Faure. This is an open-access article distributed under the terms of the Creative Commons Attribution License (CC BY). The use, distribution and reproduction in other forums is permitted, provided the original author(s) or licensor are credited and that the original publication in this journal is cited, in accordance with accepted academic practice. No use, distribution or reproduction is permitted which does not comply with these terms. 